\title{
The Sociology of Healthcare Safety and Quality
}

\author{
D Allen, J Braithwaite, J Sandall and J Waring
}

This Monograph attracts the reviewer in a number of ways.

The immediate attraction is because it is about an important global issue, that of healthcare safety and quality. The topic is important for all health professionals but is doubly so for those of us who manage or lead health services. Greater accountability for safety and quality is being required of Australian health managers in 2017 as new provisions of the Australian Healthcare Agreements' between the Commonwealth and States and Territories are being enacted. This public policy initiative also means that those of us who teach and research and are involved in the education of health professionals, particularly health managers, need to make increased effort in this area. The Monograph goes some way to being useful to assist us in these endeavours.

Consistent with the Monograph's focus on a global challenge is that the contributors drawn together in this publication come from at least four nation states, providing us with perspectives reflecting their cultural and national perspectives and providing us with points of differences that allow us to more effectively compare and contrast what might be.

The reviewer further appreciates the Monograph because the editors and authors make a distinction about their approach to this topic from the range of other approaches in contemporary vogue. These are described as disciplinebased in medical and safety science, social psychology and human factors. They emphasise that healthcare safety and quality 'cuts across many sociological concerns' that they believe have been neglected by mainstream policy and research, until recent times. The reviewer agrees with that view. There is much less sociology taught these days in health management programs than in previous times and we are the poorer for it.

\section{Edited by:}

\section{Davina Allen, Jeffrey Braithwaite, Jane Sandall and Justin Waring}

Sociology of Health and IIIness Monograph series Published by WILEY Blackwell
There is a lack of understanding by some, that we are in the people business, 'people interacting with other people'. There is increasing evidence that a lot of what we do is based on mechanistic organisational approaches and clinical reform actually adds to the degree of complication and possibly does more harm than good. There is evidence to support these assertions in the current editorial of the Journal in which this review takes place. So we agree with the editors and authors that our approach needs to be 'shaped by wider socio-cultural and political structures'.

So the editors emphasise that if healthcare safety and quality are to be understood and more effectively addressed we need to bring to the fore the importance of sociological perspectives presented in this Monograph. The Editors set out to achieve this endeavor by asking us some important questions about how to better reflect on the 'contribution of sociology and sociological engagement' so that we can better address perspectives of patient safety and quality.

These questions are:

1. How can we understand and explain the social, cultural and lived experiences of quality and safety?

2. What theories, models and concepts are useful in progressing the quality and safety agenda?

3. What is an appropriate balance between sociology 'of' and sociology 'for' quality and safety?

4. What distinctively sociological research approaches might be applied to the study of quality and safety?

5. What analytical perspectives might offer novel insights?

The editors then continue to put some structure around the following chapters. They do so using headings such as 'parallel paths, organizing, technologies and practice, concretization, culture, politics, theory and practice'. Then the editors allow the contributors to tell their stories and they all do so with a magnificent effort of weaving contemporary sociological theories and practice together with contemporary research about real healthcare issues and organizational practice. As the readership of this Journal is predominantly managers I can assure them that the language of management is used and addressed throughout. 
Policy and practice, accountability, corporate governance, sensemaking, knowledge sharing to name a few words are all there and addressed within the chapters. If you have a more clinical bent then your interests are also addressed: patient safety, acute illness, patient categorisation, primary medical care, general practice, infections and clinician accounts are all part of the language used and the topics addressed.

On reflection, this Monograph would sit well in a health manager's set of reference books. It is a Monograph that you can read in its entirety but I suspect that most might read their preferred chapters and then come back to them and other chapters over time. It is the sort of Monograph that you will access to seek inspiration or examples of approaches to reform that you can then assess the suitability of in addressing your more immediate challenges. I suspect that managers and practitioners who are really interested in better organisational and management practice or who might just want to make sense of what is happening around them will find this Monograph helpful.

At the health management education and teaching level, I suspect academics will soon discover the contents of the Monograph to be helpful for teaching across health management subjects while at the same time increasing the understanding of students in sociological theory and perspectives. The reviewer also suggests that the content provides the opportunity to develop case studies from the contents to enhance teaching practice.

Congratulations to the editors and contributing authors for this contribution of knowledge and learning relevant to the needs of those of us engaged in health systems as managers, educators, researchers and reformists.

\section{Dr DS Briggs}

Reviewer 